\title{
Kann mit Erziehungsplänen erzogen werden?
}

\section{Erziehungspläne als Verfahren zur Normalisierung von Kindern und Jugendlichen}

Stufenpläne und Verstärkerpläne sind in den Hilfen zur Erziehung mittlerweile weitverbreitete Verfahren zur "pädagogischen" Arbeit mit Kindern und Jugendlichen, insbesondere für Kinder und Jugendliche mit einem intensiven pädagogischen Bedarf. Solche Verfahren werden hier näher beleuchtet und kritisch reflektiert, wobei auch die Frage nach den positiven Effekten entsprechender Pläne gestellt wird. Immerhin ist davon auszugehen, dass pädagogische Fachkräfte vor allem auf Verfahren zurückgreifen, die einen Mehrwert für ihre Arbeit darstellen.

1 rziehungspläne sind insbesondere in der stationären Kinder- und Jugendhilfe, insbesondere in intensivpädagogischen Maßnahmen, mittlerweile ein weit verbreitetes, erzieherisch gedachtes Verfahren, um auf den Alltag der Kinder und Jugendlichen, ihre Lebenswelt und damit auf ihre weitere Entwicklung einzuwirken. Zentrale Funktion der Pläne ist, eine über Gruppenregeln und von den Pädagog_innen vertretene Normen und Werte unbestimmte „Normalität“ im Alltag der jeweiligen Wohngruppe zu erreichen. Die definitorische Setzung von Normen und Werten erfolgt hierbei in aller Regel aus einem pädagogisch-moralisch als gut verstandenen Impetus, der dabei gezwungenerweise für die Jugendlichen fremdbestimmt ist.

Erziehungspläne als Verfahren stehen dabei mindestens dem Bild einer familienanalogen Normalität in Wohngruppen entgegen. Immerhin stehen sie in einem maxi-

Mischa Engelbracht
Universität Erfurt,
Erfurt, Deutschland
*1987. Dr., Akademischer Rat an der Universität Erfurt.
Vertretungsprofessor für Sozialpädagogik und ihre Didaktik an
der Bergischen Universität Wuppertal.
mischa.engelbracht@uni-erfurt.de

Zusammenfassung Stufen- oder Verstärkerpläne erfahren eine breite Anwendung in der stationären Kinder- und Jugendhilfe und werden, teilweise medial gestützt, zu besonders wirksamen pädagogischen Verfahrensweisen erhoben. Der Beitrag setzt sich aus verschiedenen Perspektiven kritisch mit den Funktions- und Wirkungsweisen von Erziehungsplänen auseinander.

Schlüsselwörter Erziehungspläne, Stufenpläne, Verstärkerpläne, Ethnografie, Kinder- und Jugendhilfe, Stationäre Erziehungshilfe, Hilfen zur Erziehung malen Widerspruch zur privat hervorgebrachten, familiären Erziehung. Eine Erziehung, die oft gekennzeichnet ist durch die besonders ausgeprägte Beziehung (positiv wie negativ) der Familienmitglieder untereinander. Aus gesellschaftlichen Normen heraus besteht für die Jugendlichen die durchaus relevante Erwartung, dass Familien - komme was wolle und bis zum Tod der Mitglieder (möglicherweise sogar darüber hinaus) - auch über eine räumliche Distanz hinweg bestehen und in irgendeiner Form zusammengehören. Über ein in dieser Verbindlichkeit sozial konstruiertes System verfügt die professionelle Kinder- und Jugendhilfe nicht. Sie ist wesentlich flexibler und fluide, was sich auch in den zur Erziehung genutzten Verfahrensweisen widerspiegelt. Mit Verweis auf Klaus Wolf (1999) lässt sich festhalten, dass Familien auf andere Machtquellen, etwa in der Beziehung untereinander, zurückgreifen können als die Kinder- und Jugendhilfe.

Die durch Erziehungspläne angestrebte Normalität bezieht sich demnach mehr auf ein als „normal“ definiertes Handeln der Kinder und Jugendlichen im Alltag der Wohngruppen. Die Vorstellung von normalem Handeln in Form eines Erziehungszieles ist durch die Normen und Wertvorstellungen primär der pädagogischen Fachkräfte, aber auch etwa des Trägers geprägt. Neben diesen, teils heterogenen Wertvorstellungen sind es insbesondere die genutzten Verfahren, die sich auf den Alltag der Kinder und Jugendlichen auswirken. Der Beitrag beleuchtet daher spezifische Verfahrensweisen, welche in Form von Erziehungsplänen umgesetzt werden, näher.

\section{Über Erziehungspläne}

Erziehungspläne bezeichnen methodische Verfahrensweisen, die über ein normiertes Regelsystem den Gruppenalltag von Kindern, Jugendlichen und Pädagog_innen strukturieren sollen. Sie haben das Ziel, vor- 
hersehbare und damit transparente Reaktionen der pädagogischen Fachkräfte sowie der Teams auf entweder erwünschtes Handeln oder unerwünschtes Handeln festzulegen. Hierbei kann man zwischen zwei Formen von Erziehungsplänen unterscheiden:

- Verstärkerpläne und

- Stufenpläne (Engelbracht 2019).

Die Erziehungspläne, Token-Systeme, Verstärkerplane oder Stufenpläne sind aus der Verhaltenstherapie entlehnte Konditionierungssysteme (Linderkamp 2009, S. 210 ff., 215), die das Ziel verfolgen, mittels Belohnungen, Bonifikationen, Strafen, Sanktionen und über eine engmaschige Reglementierung das soziale Handeln von Kindern und Jugendlichen in deren Alltag zu steuern. Verstärkerpläne nutzen Punkte, Token, Coins, Sticker oder ähnliches, die als Gradmesser für gutes Benehmen dienen und das Erreichen eigener und fremdbestimmter Ziele belohnen. Stufenpläne ermöglichen auf der anderen Seite den Kindern und Jugendlichen unterschiedliche Bonifikationen - bisweilen auch Freiheitsgrade, die durch gutes Benehmen errungen und durch schlechtes Benehmen wieder verloren werden können.

Jede Wohngruppe entwickelt dabei eine eigene, auf ihre Bedürfnisse abzielende Form der Erziehungspläne. So kann ein Verstärkerplan beispielsweise vorsehen, dass pro Tag 20 Punkte von den Kindern und Jugendlichen erworben werden können. Im Verlauf einer Woche ist so, bei maximaler Ausbeute, der Erwerb von 140 Punkten möglich. Die erworbenen Punkte werden gesammelt, transparent öffentlich ausgehängt und können von den Heranwachsenden für Bonifikationen eingelöst werden. Im beobachteten Verstärkerplan waren es beispielsweise 140 Punkte für eine Woche gutes Benehmen, die für Süßes oder Saures, was etwa einem zusätzlichen Schokoriegel am Sonntagabend entspricht, eingelöst werden konnten. Für 350 Punkte war es möglich, eine Stunde länger eine Spielekonsole zu nutzen; für 500 eine DVD zusätzlich auszuleihen. Maximal können 3500 Punkte für den Besuch in einem Freizeitpark eingelöst werden. Dies entspricht umgerechnet, bei maximaler täglicher Ausbeute, dem Gegenwert von sechs Monaten guten, normativ angepassten Benehmens. Erwünschtes oder positiv konnotiertes Verhalten - oft als normal definiert - erhält so einen Wert, welcher als Coins oder Punkte angespart werden kann und einzig zur Bezahlung eines Bonus als Gegenwert eingesetzt werden kann. Bei einem Stufenplanmodell können die Kinder und Jugendlichen von Stufe zu Stufe aufsteigen. Sie durchlaufen dabei in der Regel alle vorgesehenen Stufen, die sowohl im Grad der durch sie erworbenen Freiheiten als auch im Grad der an die Kinder und Jugendlichen ge- stellten Anforderungen aufeinander aufbauen. Ein Stufenaufstieg ist durch das Einhalten der vorab transparent gemachten Regeln und deren Überprüfung durch die Pädagog_innen möglich. Der Verlust einer Stufe durch Abstufung erfolgt dabei durch Regelverstöße, also aufgrund nicht mehr als ausreichend konform angesehenes Handeln. Anders als beim Verstärkerplan gibt es hier also kein Kapitalpolster, sondern eine direkte Reaktion ohne Sicherungssystem auf das als abweichend eingestufte Verhalten. Der Lohn für Benehmen ist hier das Zuerkennen von Freiheiten oder Erlaubnissen, die „normalen“ Kindern und Jugendlichen, die nicht in der Einrichtung sind, bereits zusteht. Die Kinder und Jugendlichen müssen also den Nachweis erbringen, dass sie mit „normalen“ Freiheiten umgehen können.

Beide Formen von Erziehungsplänen zielen auf das soziale Handeln im Alltag der Wohngruppen und intendieren, auf die Kinder und Jugendlichen einzuwirken. Friedrich Linderkamp (2009) beschreibt etwa im Lehrbuch der Verhaltenstherapie Verstärkerpläne als ein operantes Verfahren der Psychotherapie. Grundannahme für operante Verfahren ist dabei, das neues oder erwünschtes Verhalten über Belohnungen respektive Verstärkungen bewirkt werden könne. Positive Erfahrungen, also Verstärkungen (Bonifikationen) oder Stufenaufstieg, führen, so die zugrunde gelegte Theorie, dazu, dass Kinder und Jugendliche zu entsprechend positiven und als „normal“ verstandenem Handeln angeregt werden. Operante Methoden setzen, so Linderkamp (2009), anders als die klassische Konditionierung, aktives Handeln einer Person voraus. Ohne auf primäre oder sekundäre Verstärker, Belohnungen und Bestrafungen oder unterschiedliche Verstärkerarten genauer einzugehen, bleibt festzuhalten, dass unterschiedliche therapeutische Voraussetzungen für das erfolgreiche Wirken erfüllt sein müssen. Dies ist etwa, dass erwünschtes Verhalten durch die Erziehungspläne in Form von Bonifikationen oder Stufenaufstiegen stärker belohnt werden als das Ausbleiben des erwünschten Verhaltens. Sprich: es müsste die gewaltfreie Lösung eines Konfliktes stärker belohnt werden als es etwa bei einer Schlägerei der Fall gewesen wäre. Wobei der Wert der Bonifikation, in diesem Fall etwa der Stufe, der subjektiven Einschätzung jedes einzelnen Kindes oder Jugendlichen individuell unterliegt. Dass entsprechende Erziehungspläne auf den Alltag und die Lebenswelt der Kinder und Jugendlichen einwirken und Einfluss auf die Entwicklung dieser nehmen, scheint weitestgehend unumstritten. Während im Diskurs um Erziehungspläne und engmaschige Regelsysteme die eine Seite die Wirksamkeit und Transparenz entsprechender Pläne lobt, sieht die andere Seite eine unzulässige Dressur der Kinder und Jugendlichen, die dem eigentlich in den 


\section{Extrablick: Normen in der Sozialen Arbeit}

Sozialpädagogik konsensualen Prinzip der Erziehung zur Mündigkeit entgegensteht (vgl. Lindenberg und Tilmann 2019, S. 199; Magyar-Haase 2019, S. 208 ff.).

Für eine Analyse der entsprechenden Thesen zur Wirkung von Erziehungsplänen in der Kinder- und Jugendhilfe auf das soziale Handeln beziehe ich mich auf Erkenntnisse aus der ethnografischen Feldforschung (vgl. Engelbracht 2019; Engelbracht und Leissenberger 2019). Ethnografische Forschung ermöglicht das Analysieren und Verstehen von sozialem Handeln in Praxis auch an praxisfernen Orten, wie etwa im Artikel zum Fall Niko (vgl. Engelbracht 2021) dezidierter ausgearbeitet worden ist.

\section{Ziele von Erziehungsplänen}

Die Erziehungspläne sollen also das soziale Handeln beeinflussen und zu alternativen Bewältigungsformen von Krisen und Konflikten führen. Das streng reglementierte und bürokratisch orientierte Abarbeiten einer krisenhaften Situation, etwa eines Konfliktes, wird so entkoppelt von der eigentlich pädagogisch zu bewältigenden Handlung. Die automatisierten Konsequenzen eines Tatbestandes lassen die Individuen so zu Fällen der verwalteten Erziehungspläne werden. Mohr et al. (2017) sprechen hier von organisationsbedingtem Zwang. In der pädagogischen Ko-Produktion des erzieherischen Settings laufen dabei sowohl Pädagog_innen als auch die Kinder und Jugendlichen Gefahr, dass sie als Person mit ihrer Persönlichkeit, Handlungsgründen und Emotionen für die eigentliche pädagogische Reaktion überflüssig werden. Es geht, ganz im Sinne einer judikativen Rechtsprechung, um die Tat, das Handeln, aber nicht um die beteiligten Individuen (Kinder, Jugendliche und Fachkräfte) in persona.

Kinder und Jugendliche können so die zu erwartende Konsequenz aus dem eigenen sozialen Handeln als Bestandteil eines zweckrationalen Entscheidungsprozesse gleich mit kalkulieren. Die Vorbedingung der Verstärkung durch die Erziehungspläne im Sinn einer fast exklusiven, aber starken positiven Verstärkung kann allerdings nicht in der Kinder- und Jugendhilfe erreicht werden. Immerhin ist die Akzeptanz von erzieherischen Maßnahmen durch die Jugendlichen - etwa im Sinne eines Eigenantriebs - grundsätzlich nicht voraussetzbar, noch sind Einrichtungen der Kinder- und Jugendhilfe keine in sich geschlossenen Systeme. Es bestehen in allen Einrichtungen nicht nur auf die Jugendlichen einwirkende Außenbeziehungen über Schule, Familie, Praktika etc., sondern auch die Gruppe der Kinder und Jugendlichen verfügt über eigene Logiken sozialen Handelns und damit eigene Verstärkungslogiken im Alltag. So hat, um das Gesicht bei Konflikten zu wahren, nicht einmal der längere Verzicht auf das eigene Smartphone (aufgrund der unweigerlichen Rückstufung) einen ausreichend hohen Stellenwert, um einem Konflikt aus dem Weg zu gehen oder diesen ohne Gewalt zu lösen. Das von der Einrichtung als abweichend definierte Handeln, kann von den Jugendlichen mit den zu erwartenden Konsequenzen bei Handlungsentscheidungen gleich mit ,eingepreist“" werden.

Die erworbenen Punkte oder ein Stufenaufstieg werden so zu einem Nebeneffekt, der quasi im Vorbeigehen erzielt wird. Der Punktgewinn oder Stufenaufstieg erfolgt also trotz des individuellen Handelns und nicht wegen der Option, sich positive Effekte zu verdienen. Mögliche erfolgreiche Anpassungsleistungen der Jugendlichen lassen sich kaum auf die Erziehungspläne zurückführen, auch weil eine Abgrenzung von weiteren und anderen pädagogischen Einflüssen kaum möglich ist. Die Wirkung von Erziehungsplänen auf die Kinder und Jugendlichen scheint also überschaubar zu sein.

So gibt es Jugendliche, die entsprechende Systeme als „ungerechtfertigte Zumutung“, als „ambivalente Herausforderung “ oder aber als "unbedeutenden Nebenschauplatz“ (Gaßmöller 2019, S. 294) empfinden. Besonders interessant ist die Rückschau der Jugendlichen und jungen Erwachsenen auf die Wirksamkeit der entsprechenden Regelsysteme. Wirkung meint dabei, ob das durch das System intendierte Ziel eingetreten ist. Es ist davon auszugehen, dass weder die Rebellen, welche die Einrichtung und ihre Regeln ablehnen, noch die Rationalen (Menk, Schnorr und Schrapper 2013, S. 65 ff.), die eine Orientierung an den Regeln nur vortäuschen, etwa als „Bluffer“ oder aber das System an sich für ihre Zwecke zu nutzen wissen, besonders viele Werte und Normen für sich übernommen haben. Der dritte Typus, der das Regelsystem akzeptiert, ist der Typus, der auch andere Regelsysteme eher akzeptiert, auch weil der engmaschige Erziehungsplan als unverhältnismäßig, mindestens jedoch fragwürdig empfunden wird. In gewisser Weise wirkt es doch zumindest beruhigend, dass sich Jugendliche im Allgemeinen und noch weniger die als erziehungsresistent gelabelten konditionieren lassen würden, sondern eigene Bewältigungskompetenzen für die von der Kinder- und Jugendhilfe hervorgebrachten - auch positiven oder produktiven - Krisen entwickeln. Wenn dies auch nicht unbedingt immer pädagogisch intendiert ist. Die bereits implizit erwähnte Bürokratisierung des erzieherischen Handelns hat einen besonderen Effekt. Die für die pädagogische Arbeit wichtige Ko-Produktion des Settings, aber auch die Interaktion und Beziehungsarbeit werden durch die Regelsysteme beeinflusst. Das Risiko, welches dabei in verschiedenen Einrichtungen herausgearbeitet werden konnte (Engelbracht 2019), besteht in 
der sich reduzierenden Kommunikation um das Fehlverhalten. Es scheint einfach, nur auf Regeln verweisen zu müssen, wo die folgende Sanktion auch bereits einheitlich für alle vorab ersichtlich ist. Oder etwas bildhafter ausgedrückt, es ist arbeitsökonomisch einfacher, einen Aushang an die Küchentür zu hängen und im Zweifel auf diesen zu verweisen, als mit den einzelnen Jugendlichen immer wieder aufs Neue verhandeln - es ist einfacher, den Strom in einem Zimmer einfach auszuschalten, als tagelang zu diskutieren, was Zimmerlautstärke heißt. Auch wenn diese Diskussionen und Auseinandersetzungen mit zu den anstrengendsten in der Arbeit zählen, sind auch sie pädagogisch nicht nur notwendig, sondern möglicherweise auch die wertvollsten.

\section{Über die Funktion von Erziehungsplänen im Alltag}

Trotz der offenen Fragen und Kritikpunkte lässt sich feststellen, dass eine Vielzahl von Einrichtungen heute mit Erziehungsplänen arbeitet. Es ist also naheliegend, dass sich pädagogische Fachkräfte eine positive Wirkung aus entsprechenden Verfahrensweisen erhoffen oder aber auch selbst auf positive Erfahrungen zurückblicken können. Unbeleuchtet bleibt zumeist, dass Erziehungspläne in Anlehnung an Klaus Wolf (1999) eine depersonalisierte Machtquelle darstellen, die auf bürokratischen und damit nicht individuellen Entscheidungsgrundlagen beruht. Auch dann, wenn auf pädagogischen Gründen von den festgeschriebenen Regeln abgewichen wird, da die Heranwachsenden auf diese Abweichungen keinen Anspruch haben. Ein einmal etabliertes akzeptiertes Regelsystem innerhalb der Einrichtungskultur stellt eine nicht unerhebliche Machtquelle für die Pädagog innen dar (Engelbracht 2019) dar. Hieran anschließend ist auch die für die Kinder und Jugendlichen gegebene Transparenz ein positiv diskutierter Grund. Ein Argument, das sowohl von den Adressat_innen als auch den pädagogischen Fachkräften wiederholt geäußert wird.

Ein positiver Effekt lässt sich über einen tiefergehenden Blick in die einzelnen Bestandteile des Verfahrens herausarbeiten. Wie erläutert, unterscheiden sich von Einrichtung zu Einrichtung die verschiedenen Pläne signifikant. Im Fall einer untersuchten Wohngruppe mit Verstärkerplan scheinen gerade, als zwingender Bestandteil des Verstärkerplans, die allabendlichen Gespräche unter vier Augen, in denen strukturiert der Tag reflektiert, Wünsche und Kritik geäußert, aber auch Pläne für den kommenden Tag und die Woche geschmiedet werden, bei den Kindern und Jugendlichen eine Wirkung auf alltägliche Entscheidungsprozesse und die Beziehungsgestaltung zu den Pädagog_innen zu haben. Dabei haben sich die Jugendlichen bereits den Tag über vorgenommen, verschiedene Themen mit in die Refle- xion zu nehmen und auch Kritik an Entscheidungen zu üben und „es ihnen zu sagen“. Der in den Gesprächen reglementarisch vor negativen Konsequenzen geschützte und von akuten Konflikten handlungsentlastete Rahmen bietet so die Gelegenheit, auch Entscheidungen zu revidieren und die Perspektive der jeweiligen anderen Seite kennen zu lernen (vgl. Engelbracht 2019). Ebenso wirken die Regeln und ihre Anforderungen auch auf den Alltag der Pädagog_innen in den Wohngruppen. Immerhin sind alle Beteiligten, bei entsprechender Ausgestaltung, gezwungen, sich für die alltäglichen individuellen Gespräche am Abend die Zeit zu nehmen und diese umzusetzen. Das Verfahren der Erziehungspläne und die Umsetzung dieser wirken sich also auch auf die Priorisierung der pädagogischen Arbeit mit den Kindern und Jugendlichen aus. Die abendlichen Gespräche sind als Ritual fest in den Erziehungsplänen fixiert und können nicht verschoben werden. Kein Konflikt und keine Krise in der Wohngruppe haben hier einen Einfluss drauf. Indizien für einen weiteren positiven Effekt finden sich in den Äußerungen der pädagogischen Fachkräfte. Klare Regeln und Strukturvorgaben geben Sicherheit bei pädagogischen Entscheidungen. Insbesondere Kolleg_ innen mit weniger Berufserfahrung scheinen die so gegebene Klarheit im pädagogischen Alltag zu schätzen. Die Erziehungspläne übernehmen so die Funktion einer strukturiert festgelegten Teamleitung. Fachkräfte, die neu in einer Wohngruppe anfangen, haben so von Anfang an ein Regel-, Reaktions-, und Sanktionswerk, das Sicherheit im Alltag der Wohngruppe vermittelt.

\section{Ausblick}

Die Diskussion suchte das pädagogische Spannungsfeld, das sich zwischen den auf der einen Seite individuell auszuhandelnden und auf der anderen Seite institutionellen Regeln sichtbar zu machen. Je mehr Regeln institutionalisiert vorgegeben sind, umso weniger individuelle Interaktion ist notwendig beziehungsweise umso weniger Raum wird es für diese geben. Unwidersprochen bleibt dabei der Einwurf, dass tägliche Aushandlungs- und Entscheidungsprozesse (auch) eine Zumutung für die Beteiligten bedeuten. Ein in der Erziehungswissenschaft immer wieder diskutiertes Paradox (Helsper 2012).

In Bezug auf die Frage, ob und wie Erziehungspläne eine Wirkung auf Kindern und Jugendlichen entfalten können, zeigen sich die Befunde eher ambivalent bis kritisch. Einen stärkeren Einfluss scheinen Erziehungspläne aber auf das Agieren der Pädagog_innen zu haben. So konnte herausgearbeitet werden, dass Erziehungspläne nicht nur für unerfahrene Fachkräfte eine Alltagsentlastung und Sicherheit bieten können, son- 


\section{Extrablick: Normen in der Sozialen Arbeit}

dern auch einen Einfluss auf das direkte pädagogische Handeln haben können.

Eingegangen. 1. März 2021

Angenommen. 29. März 2021

Funding. Open Access funding enabled and organized by Projekt DEAL.

Open Access. Dieser Artikel wird unter der Creative Commons Namensnennung 4.0 International Lizenz veröffentlicht, welche die Nutzung, Vervielfältigung, Bearbeitung, Verbreitung und Wiedergabe in jeglichem Medium und Format erlaubt, sofern Sie den/die ursprünglichen Autor(en) und die Quelle ordnungsgemäß nennen, einen Link zur Creative Commons Lizenz beifügen und angeben, ob Änderungen vorgenommen wurden.

Die in diesem Artikel enthaltenen Bilder und sonstiges Drittmaterial unterliegen ebenfalls der genannten Creative Commons Lizenz, sofern sich aus der Abbildungslegende nichts anderes ergibt. Sofern das betreffende Material nicht unter der genannten Creative Commons Lizenz steht und die betreffende Handlung nicht nach gesetzlichen Vorschriften erlaubt ist, ist für die oben aufgeführten Weiterverwendungen des Materials die Einwilligung des jeweiligen Rechteinhabers einzuholen.

Weitere Details zur Lizenz entnehmen Sie bitte der Lizenzinformation auf http://creativecommons.org/licenses/by/4.0/deed.de.

\section{Literatur}

Engelbracht, M. (2019). Jugendliches Alltagsleben in freiheitsentziehenden Maßnabmen. Eine rekonstruktive Studie über sozialpädagogische Erziehungsprozesse bei Jugendlichen mit multikomplexen Risikolagen. Wiesbaden: VS.

Engelbracht, M. (2021). Krisenbewältigung von Pädagog*innen im Umgang mit aggressiven Kindern und Jugendlichen. Ethnografische Rekonstruktion eines Konfliktes oder ,der Fall Niko‘. In M. Dörr \& B. Ahrbeck (Hrsg.), Jugendkriminalität - Diskurse, Verstehen und (psychoanalytisch) pädagogische Antworten. Gießen: Psychosozial Verlag.

Engelbracht, M., \& Leissenberger, F. (2019). Teilhabe trotz Sozialpädagogik? Partizipation und freiheitsentziehende Maßnahmen. In Kommission Sozialpädagogik (Hrsg.), Teilhabe durch"in*trotz Sozialpädagogik (S. 132145). Weinheim \& Basel: Beltz Juventa.

Gaßmöller, A. (2019). Volle Punktzahl für gutes Benehmen?! Verstärkersysteme in stationären Maßnahmen der Kinder- und Jugendhilfe. In DGSA (Hrsg.), Soziale Arbeit in der Demokratie - Demokratieförderung in der Sozialen Arbeit (S. 289-300). Opladen \& Farmington Hills: Barbara Budrich.

Helsper, W. (2012). Antinomien der Moderne in Einführung. In H.-H. Krüger \& W. Helsper (Hrsg.), Einführung in die Grundbegriffe und Grundfragen der Erziehungswissenschaft (S. 15-34). Opladen \& Farmington Hills: Barbara Budrich.

Lindenberg, M., \& Tilman, L. (2019). Zwang und Erziehung. Forum Erziehungshilfen, 25(4), 199-203.

Linderkamp, F. (2009). Operante Methoden. In S. Schneider \& J. Margraf (Hrsg.), Störungen im Kindes- und Jugendalter. Lehrbuch der Verhaltenstherapie, (Bd. 3, S. 209-220). Heidelberg: Springer.

Magyar-Haas, V. (2019). Beschämende Aspekte verhaltenstherapeutischer Stufenpläne. Forum Erziehungshilfen, 25(4), 208-212.

Menk, S., Schnorr, V. \& Schrapper, C. (2013). „, Woher die Freiheit bei all dem Zwange?" Langzeitstudie zu (Aus-) Wirkungen geschlossener Unterbringungen in der Jugendhilfe. Weinheim und Basel: Beltz Juventa.

Mohr, S., Ritter, B., \& Ziegler, H. (2017). Zwang als erzieherisches Mittel in der Kinder- und Jugendhilfe? Sozial Extra. https://doi.org/10.1007/s12054017-0082.

Wolf, K. (1999). Machtprozesse in der Heimerziehung. Eine qualitative Studie über ein Setting klassischer Heimerziehung. Münster: Votum.

Hier steht eine Anzeige.

算 Springer 